\title{
ON THE DISCONTINUOUS FLOW AROUND AN AIRFOIL WITH FLAP*
}

\author{
BY \\ P. W. KETCHUM \\ University of Illinois**
}

Summary. A method is given for calculating the effect of the wake on the pressure distribution produced by the steady, two dimensional flow around an airfoil with infinite span and broken line profile. To determine the size of the wake there is proposed the hypothesis that the flow is such as to create a minimum disturbance at distant points.

1. Introduction. The general Navier-Stokes equations for the steady, viscous flow of an incompressible fluid around an immersed body have not been solved even for such simple cases as the sphere or cylinder. However, it is well known that certain aspects of the flow are adequately represented by neglecting viscosity entirely. Thus the perfect fluid theory of Kutta and Joukowski for calculating the reaction of the air on an airfoil with infinite span, engaged in a uniform rectilinear translation through a bulk of air at rest, gives satisfactory results so far as the lift is concerned. This theory fails to account for all the details of the flow, however. In particular, it neglects the wake of deadwater which exists at the trailing edge. The effect of this wake is to make the lift slightly smaller than the calculated value and to reduce the negative pressures along the rear portion of the suction side. Since the effect is greatest near the trailing edge, the discrepancies between calculations and observation will be most pronounced for the total hinge moment of the pressures on a trailing edge flap.

The boundary layer theory has been used to calculate the size of the wake, i.e. the point where the wake detaches itself from the body. Basing his calculations on the observed pressure distribution, Hiemenz in 1911 obtained from the boundary layer theory an angle of $82^{\circ}$ from the forward stagnation point for the point of detachment in flow around an infinite circular cylinder, a value in good agreement with his experiments. However, his experiments were made at low Reynolds numbers; an airfoil would be stalled for such a flow. For the high Reynolds numbers corresponding to the normal, unstalled flight of an airfoil, the calculated point of detachment for flow around the cylinder is at least $20^{\circ}$ smaller than the observed value, even though the calculations are based on experimental pressure distributions.

* Received Dec. 30, 1942.

** This investigation was undertaken at the suggestion of Professor W. Prager while the author was a Fellow under the Program of Advanced Instruction and Research in Mechanics at Brown University. 
A definite advance was made by C. Schmieden in $1932^{1}$ when he showed that for high Reynolds numbers the perfect fluid theory is adequate to explain the wake behind a cylinder if one assumes a discontinuous flow. According to this view, the wake is obtained as a special case of the classical theory of jets. The theory of jets, due to Helmholtz and Levi-Civita, assumes a wake of stationary fluid at uniform pressure, bounded by two streamlines along which the speed is constant and different from zero. In spite of the fact that such a discontinuity in velocities would be impossible in a real fluid, Schmieden's calculated values of the pressures around the cylinder agree remarkably well with experiment. The agreement is perfect up to the pressure minimum; beyond that there are some deviations, which can be attributed to turbulence in the boundary layer and circulation of fluid in the wake. The point of detachment is approximately correct, but the observed point is somewhat masked by the turbulence; and the circulation of the fluid in the wake causes the constant pressure in the wake to be negative instead of zero as predicted by the theory.

Schmieden found, however, that the equations of motion for a perfect fluid plus the usual boundary condition that the velocity approach the stream velocity as the distance from the cylinder increases without bound are insufficient to determine the discontinuous flow uniquely. Various sizes and types of wakes are possible. There exist symmetric discontinuous flows with wakes detaching from the cylinder at every angle from about $55^{\circ}$ to $180^{\circ}$. For angles between $55^{\circ}$ and about $124^{\circ}$ the wake extends to infinity and is bounded by streamlines which are first concave toward the wake and then, after passing through a point of inflection, are convex; the drag on the cylinder is positive; and the constant pressure in the wake is zero. For angles between $124^{\circ}$ and $180^{\circ}$ the wake is finite in extent, the drag is zero, and the pressure in the wake is positive. ${ }^{2}$ For the critical angle of about $55^{\circ}$ the wake boundaries are always convex (Helmholtz case). For the critical angle of about $124^{\circ}$ the wake is infinite but has a width which tends asymptotically to zero, the boundaries of the wake are concave, the drag vanishes, and the pressure in the wake is zero; we shall refer to this as the Schmieden case.

Since a correctly set physical problem should have a unique answer even for a perfect fluid, an additional boundary condition is evidently required to determine the flow. Schmieden has chosen the auxiliary condition that the wake boundaries be concave and infinite in extent. A possible reason for selecting the condition of concavity is the intuitive feeling that in a viscous fluid any portion of a streamline with a point of inflection would be swept

${ }^{1}$ Schmieden, C., Über die Eindeutigkeit der Lösungen in der Theorie der unstetigen Strömungen, Ingenieur-Archiv 3, pages 356-370 (1932) and 5, 373-375 (1934).

2 Schmieden considers only infinite wakes, but it is obvious that finite wakes can exist: see Kolscher, M., Unstetige Strömungen mit endlichem Totwasser, Luftfahrtforschung 17, 154160 (1940). The present paper also restricts attention to infinite wakes. 
away to infinity. However, for a perfect fluid there would be no such tendency; moreover, the condition of concavity is an "internal" condition, more in the nature of a law of motion than of a boundary condition. The required boundary condition must be "external" in character-a condition which can be imposed arbitrarily as a constraint on the system. Such an external condition could only be imposed by specifying something about the nature of the impinging stream. In particular we could require that this stream be as nearly uniform as possible, that is, the difference between the velocity and the undisturbed stream velocity shall approach zero as rapidly as possible as one recedes from the cylinder in the direction of the stream source. In the present paper we show that this assumption of "minimum disturbance at infinity" does in fact lead to the Schmieden case, at least for infinite wakes.

On the experimental side the justification for choosing the Schmieden case for an approximation to the flow of an actual fluid at high Reynolds numbers lies in the agreement previously mentioned between calculated and observed pressure distributions. ${ }^{3}$ Of the various discontinuous flows the Schmieden case gives the best agreement for the pressure distribution as a whole, but more particularly for the forward part of the cylinder where turbulence is absent. As previously mentioned the observed pressure in the wake is negative instead of zero, but this is still the best agreement possible since the theory predicts a positive pressure for finite wakes. Quantative technical applications involving pressures behind the cylinder are subject to the limitations imposed by this discrepancy. Further refinements of the theory to eliminate this discrepancy by taking into account the turbulence in the wake have been suggested by Schmieden (Reference 1, page 364).

In $1940 \mathrm{Schmieden}^{4}$ applied his theory of discontinuous flow to the case of an airfoil with infinite span whose profile is an inclined straight line segment. It was found that the pressure distributions so obtained agree almost exactly with the pressure calculated from the Kutta-Joukowski theory except near the trailing edge, where the wake would be expected to exert an appreciable influence. It should be observed that the Kutta-Joukowski theory also uses an additional assumption beyond those involved in the perfect fluid equations, namely, the Joukowski hypothesis that the circulation is such that the velocity at the trailing edge is finite. Schmieden makes no direct assumption about the circulation, but replaces the Joukowski hypothesis by the above assumption concerning the nature of the wake. From Schmieden's point of view the Joukowski hypothesis is a special assumption concerning the nature of the wake in the limiting case when the wake is made to disappear.

${ }^{3}$ Schmieden compares his results with the experimental values of Eisner, F., Widerstandsmessungen an umströmten Zylindern, Mitteilungen der Preussischen Versuchsanstalt für Wasserbau and Schiffbau, Heft 4, Berlin 1929.

'Schmieden, C. Über Tragflïgelströmungen mit Wirbelablösung, Luftfahrtforschung 17, 37-44 (1940). 
An objection has been raised to Schmieden's theory of the airfoil with straight line profile in that the pressure is negatively infinite at the leading edge; it is one of the requirements of the classical theory of jets that separation of the fluid from the body occurs at such points. However, in an actual airfoil no separation occurs at the leading edge (unless the airfoil is stalled) because the leading edge is rounded off. The infinite velocity is not a necessary feature of the Schmieden theory, but only results from the sharp leading edge which was assumed to render the mathematics feasible. Singularities of exactly the same order are obtained from the Kutta-Joukowski theory when applied to sharp leading edges.

In the present paper we apply the Schmieden theory to the case of two dimensional flow around an airfoil with flap, the profile being a broken line. Some of the results are compared with the Kutta-Joukowski theory in Figs. 2 to 5 .

2. The general results. Consider a plate whose cross section is a broken straight line (Fig. 1). The angle of attack of the main wing may be denoted

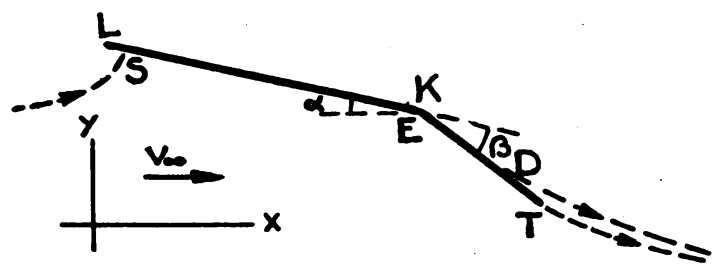

FIG. 1.

by $\alpha$ and the angle of attack of the flap by $\alpha+\beta$. Thus $\beta$ is the angle of depression of the flap. We assume that $\alpha$ is positive and that $\beta$, if negative, is greater than $-\alpha$. The velocity of the undisturbed stream (velocity of flight) is denoted by $v_{\infty}$.

There will be a critical streamline which divides at the stagnation point $S$, follows both sides of the airfoil to the points of detachment, $D$ and $T$, and then to infinity as boundaries of the wake. At the points of detachment the boundaries of the wake leave the plate tangentially.

Evidently the ratio of the lengths (chords) $\overline{K T}$ to $\overline{L K}$ must not be too small, or else the point of detachment $D$ will not fall on the flap. We restrict attention to cases where the flap is long enough for $D$ to lie on the flap-an assumption that appears to be justified in all cases of technical interest.

The method of Levi-Civita* does not give the velocity distribution along the surface of the plate directly in terms of the position which the point under consideration has on the plate; but instead, both position and velocity

* For an exposition of the Levi-Civita theory in English see: Milne-Thomson, Theoretical Hydrodynamics, London, 1938, pp. 305-316. 
are expressed parametrically in terms of an auxiliary variable $\phi$, where $\phi$ is an angle ranging from 0 to $\pi$. The explicit expressions are as follows, where $v$ is the speed of the fluid and $s$ is the distance measured along the broken line from $T$. (For the proofs of these formulas see section 5):

$v=v_{\infty} \frac{\sin \frac{1}{2}\left(\phi_{L}+\phi\right) \sin \frac{1}{2}\left|\phi_{S}-\phi\right|}{\sin \frac{1}{2}\left|\phi_{L}-\phi\right| \sin \frac{1}{2}\left(\phi_{S}+\phi\right)}\left[\frac{\sin \frac{1}{2}\left(\phi_{K}+\phi\right) \sin \frac{1}{2}\left|\phi_{E}-\phi\right|}{\sin \frac{1}{2}\left|\phi_{K}-\phi\right| \sin \frac{1}{2}\left(\phi_{E}+\phi\right)}\right]^{\beta / \pi}$

$s=4 c^{2} \int_{0}^{\phi} \frac{\sin \frac{1}{2}\left(\phi_{L}-\phi\right) \sin ^{2} \frac{1}{2}\left(\phi_{S}+\phi\right)}{\sin \frac{1}{2}\left(\phi_{L}+\phi\right)}\left[\frac{\sin \frac{1}{2}\left|\phi_{K}-\phi\right| \sin \frac{1}{2}\left(\phi_{E}+\phi\right)}{\sin \frac{1}{2}\left(\phi_{K}+\phi\right) \sin \frac{1}{2}\left|\phi_{E}-\phi\right|}\right]^{\beta / \pi} \sin \phi d \phi$.

Here the constants $\phi_{E}, \phi_{S}, \phi_{L}, \phi_{K}$ and $c^{2}$ depend on the dimensions of the plate and on $\alpha$ and $\beta$. The number $c^{2}$ is a proportionality factor /determining the scale of the figure. Values of $\phi$ between 0 and $\phi_{L}$ correspond to points on the under side of the plate while values between $\phi_{L}$ and $\pi$ give points on the upper side. The determination of the numbers $\phi_{E}, \phi_{S}, \phi_{L}$, and $\phi_{K}$ is the most difficult part of the whole procedure and will be discussed later. For the present we suppose that they are known. It will be sufficient now to observe that these four constants are angles between 0 and $\pi$, whose magnitudes are in the order written, which if used as the upper limit in (2) will yield the positions of the points $E, S, L$, and $K$ respectively. Also, the angles $\phi_{S}$ and $\phi_{L}$ are near $\pi / 2$ if $\alpha$ and $\beta$ are small.

If the integration in (2) is performed there is obtained with (1) a complete parametric representation of the speed at the airfoil surface. From Bernoulli's theorem it is then a simple matter to calculate the pressure in terms of the parameter $\phi$. If the pressure at infinity is $p_{\infty}$, the pressure at any point will be

$$
p=\frac{\rho}{2}\left(v_{\infty}^{2}-v^{2}\right)+p_{\infty}
$$

where $\rho$ is the density of the fluid.

The total hydrodynamic forces acting on all or part of the airfoil may be found by integration of the pressure. In particular, the total moment of the forces acting on the flap taken with respect to the hinge will be

$$
M_{H}=\int_{0}^{\phi_{E}} p\left(l_{F}-s\right) \frac{d s}{d \phi} d \phi-\int_{\phi_{K}}^{\pi} p\left(l_{F}-s\right) \frac{d s}{d \phi} d \phi,
$$

where $l_{F}$ is the length (chord) of the flap $\overline{E T}$ and moments in the counterclockwise direction are considered positive.

The resultant force on the airfoil is vertical; the drag vanishes and the total lift is (section 9):

$$
Y=-\frac{1}{2} \pi \rho v_{\infty}^{2} c^{2}\left[\sin 2 \phi_{L}-\sin 2 \phi_{S}+\frac{\beta}{\pi}\left(\sin 2 \phi_{K}-\sin 2 \phi_{E}\right)\right] .
$$


In addition to the distributed pressures there is a concentrated force (suction) at the leading edge which acts parallel to the plate $L K$ (see section 10). Its horizontal component must equal the horizontal component of the resultant pressure on the airfoil, since there is no concentrated force at the hinge $K$. The magnitude of the force at $L$ is

$$
F=4 \pi \rho v_{\infty}^{2} c^{2} \sin ^{2} \phi_{L} \sin ^{2} \frac{1}{2}\left(\phi_{L}-\phi_{S}\right)\left[\frac{\sin \frac{1}{2}\left(\phi_{L}+\phi_{K}\right) \sin \frac{1}{2}\left(\phi_{L}-\phi_{E}\right)}{\sin \frac{1}{2}\left(\phi_{K}-\phi_{L}\right) \sin \frac{1}{2}\left(\phi_{L}+\phi_{E}\right)}\right]^{\beta / \pi} .
$$

The integrations in (2) and (4) must in general be carried out numerically or graphically. The integral in (2) has been carried out in closed form only

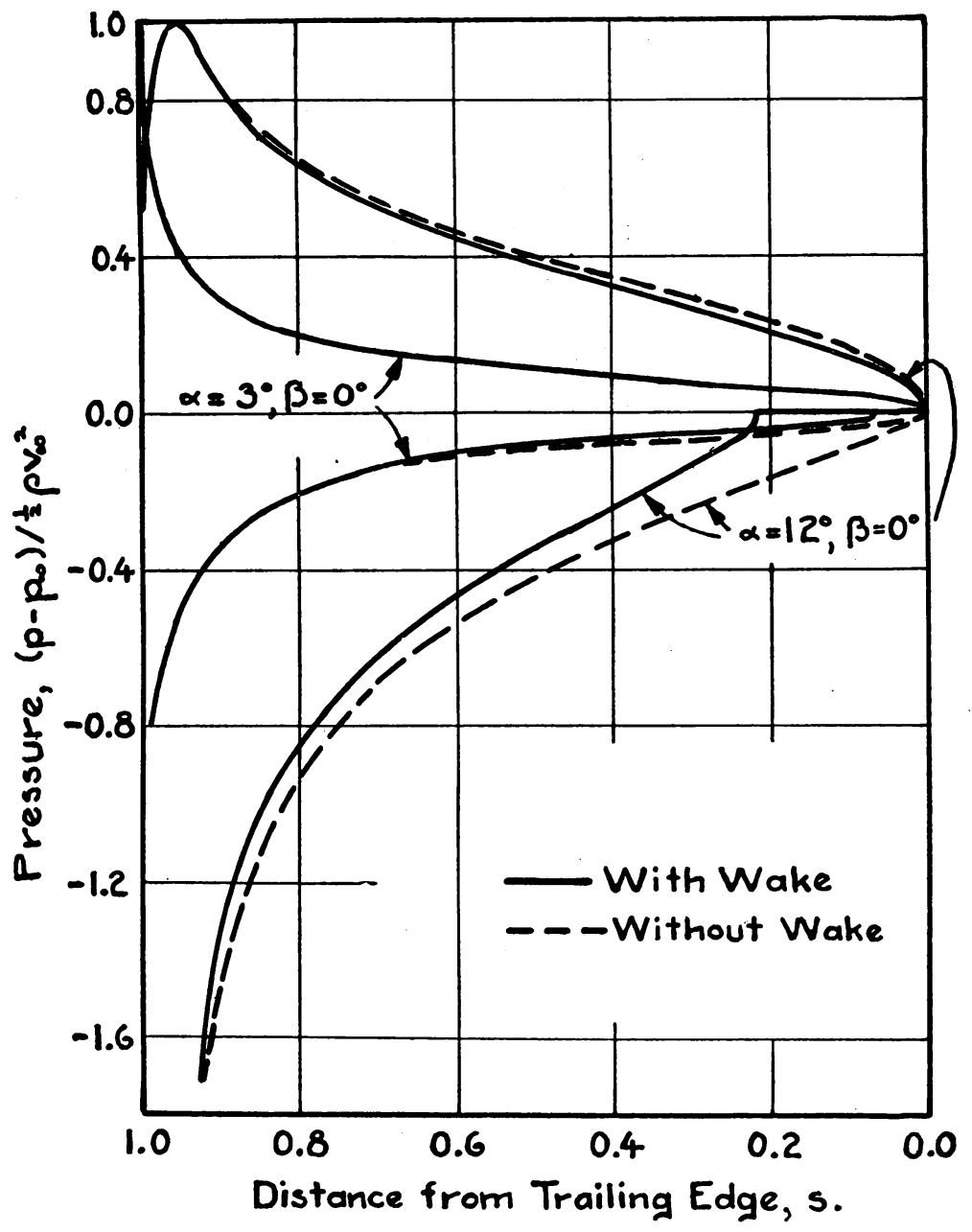

Fig. 2. 
for the case of a straight plate, i.e., $\beta=0$, (Schmieden, Reference 4 ). The problem is much simplified in that case by the fact that of the four constants, $\phi_{E}$ and $\phi_{K}$ do not occur and $\phi_{L}=\frac{1}{2}(\pi+\alpha), \phi_{S}=\frac{1}{2}(\pi-\alpha)$. (See section 4 for the proof).

3. Some numerical results. If the angle of depression of the flap is very small in comparison with the angle of attack, we consider as a first approximation that $\beta=0$. Schmieden (Reference 4 ) has given a diagram for the pressure

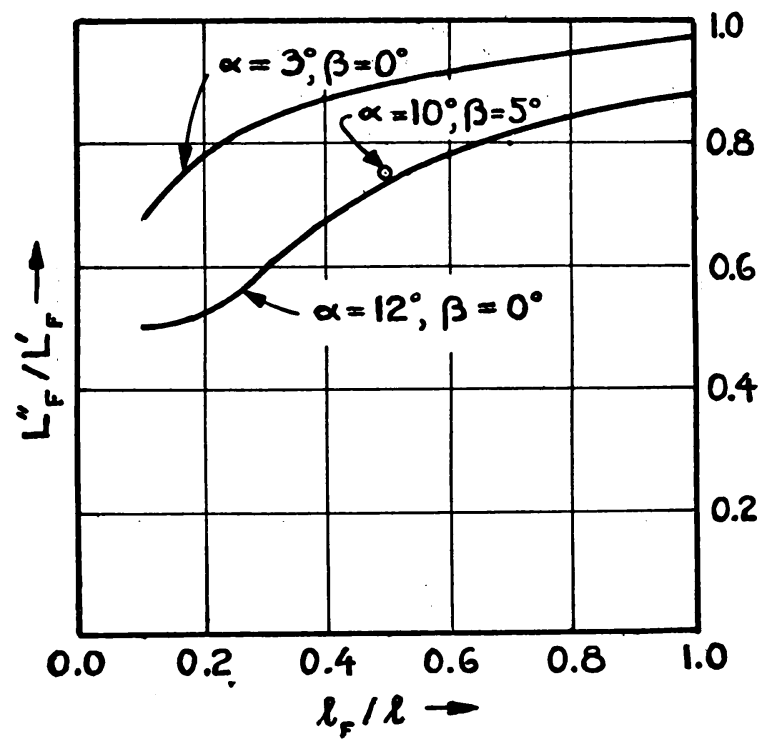

Fig. 3.

$L_{F}^{\prime}=$ Total lift on flap without wake,

$L_{F}{ }^{\prime \prime}=$ Total lift on flap with wake,

$l_{F}=$ length of flap $=\bar{K} \bar{T}$

$l=$ total length of airfoil $=\bar{L} \bar{K}+\bar{K} \bar{T}$.

distribution in this case for an angle of attack of $12^{\circ}$, showing a comparison of values obtained both with (Schmieden theory) and without (KuttaJoukowski theory) a wake. His diagram is reproduced in Fig. 2, together with a similar diagram for an angle of attack of $3^{\circ}$.

Fig. 3 shows the effect of the wake on the total lift $L_{F}$ of the flap, for flaps of various sizes. Fig. 4 is a similar diagram for the hinge moment $M_{H}$ of the flap.

Fig. 5 shows the effect of the wake on the pressure distribution for the case $\alpha=10^{\circ}, \beta=5^{\circ}$ and $\lambda=\bar{K} \bar{T} / \overline{L K}=1.00 .^{5}$

- For the Kutta-Joukowski solution see: Schmieden, C., Die Strömung um einen ebenen Tragflügel mit Querruder, Zeitschrift f. angew. Math. u. Mech. 16, 194-198 (1936).

See also Ellenberger, G., Bestimmung der Luftkräfte auf einen ebenen Tragflügel mit Querruder, Zeitschrift f. angew. Math. u. Mech. 16, 199-226 (1936). 
4. The conditions for the determination of the constants. The Schmieden hypothesis. As $\phi$ varies from 0 to $\phi_{E}$ the corresponding point in Fig. 1 will move along the under side of the flap from $T$ to $E$; as $\phi$ goes in succession from $\phi_{E}$ through $\phi_{S}, \phi_{L}, \phi_{K}$ to $\pi$, the corresponding point will move along

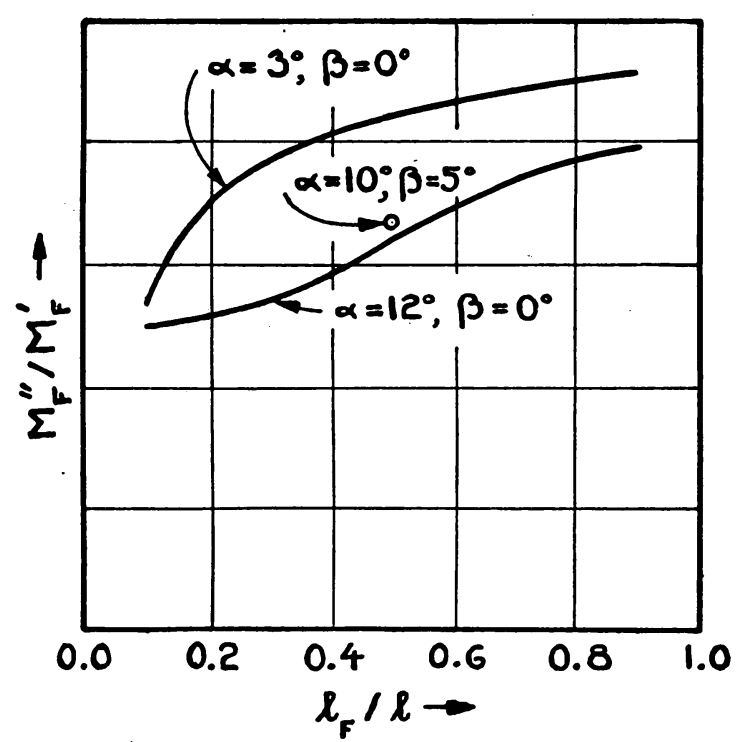

FIG. 4.

$M_{H}^{\prime}=$ Hinge moment on flap without wake, $\quad l_{F}=$ length of flap $=\bar{K} \bar{T}$,

$M_{H}{ }^{\prime \prime}=$ Hinge moment on flap with wake, $\quad l=$ total length of airfoil $=\bar{L} \bar{K}+\bar{K} \bar{T}$.

the under side of the plate through $S$ to $L$ and then back along the upper side through $K$ to $D$. Now the distance traversed on the under side between $E$ and $L$ must equal the distance on the upper side from $L$ to $K$. Hence, if the integrand in equation (2) is $f\left(\phi, \phi_{E}, \phi_{S}, \phi_{L}, \phi_{K}\right)$, then

$$
\int_{E}^{\phi_{L}} f\left(\phi, \phi_{E}, \phi_{S}, \phi_{L}, \phi_{K}\right) d \phi=\int_{\phi_{K}}^{\phi_{L}} f d \phi .
$$

If $\lambda=\overline{T K} / \bar{K} L$ is the ratio of the length of the flap to the length of the stationary part of the airfoil, we must have

$$
\int_{0}^{\phi_{E}} f d \phi=\lambda \int_{\phi_{E}}^{\phi_{L}} f d \phi
$$

Equations (5) and (6) are the two conditions on the constants imposed by the geometry of the airfoil. A third condition is obtained from the requirement that the velocity must approach the stream velocity as the distance 
from the airfoil tends to infinity. The following equation is the mathematical formulation of this condition (for the proof see section 6):

$$
\phi_{L}-\phi_{S}+\frac{\beta}{\pi}\left(\phi_{K}-\phi_{E}\right)=\alpha+\beta \text {. }
$$

For the fourth condition we need the Schmieden hypothesis mentioned

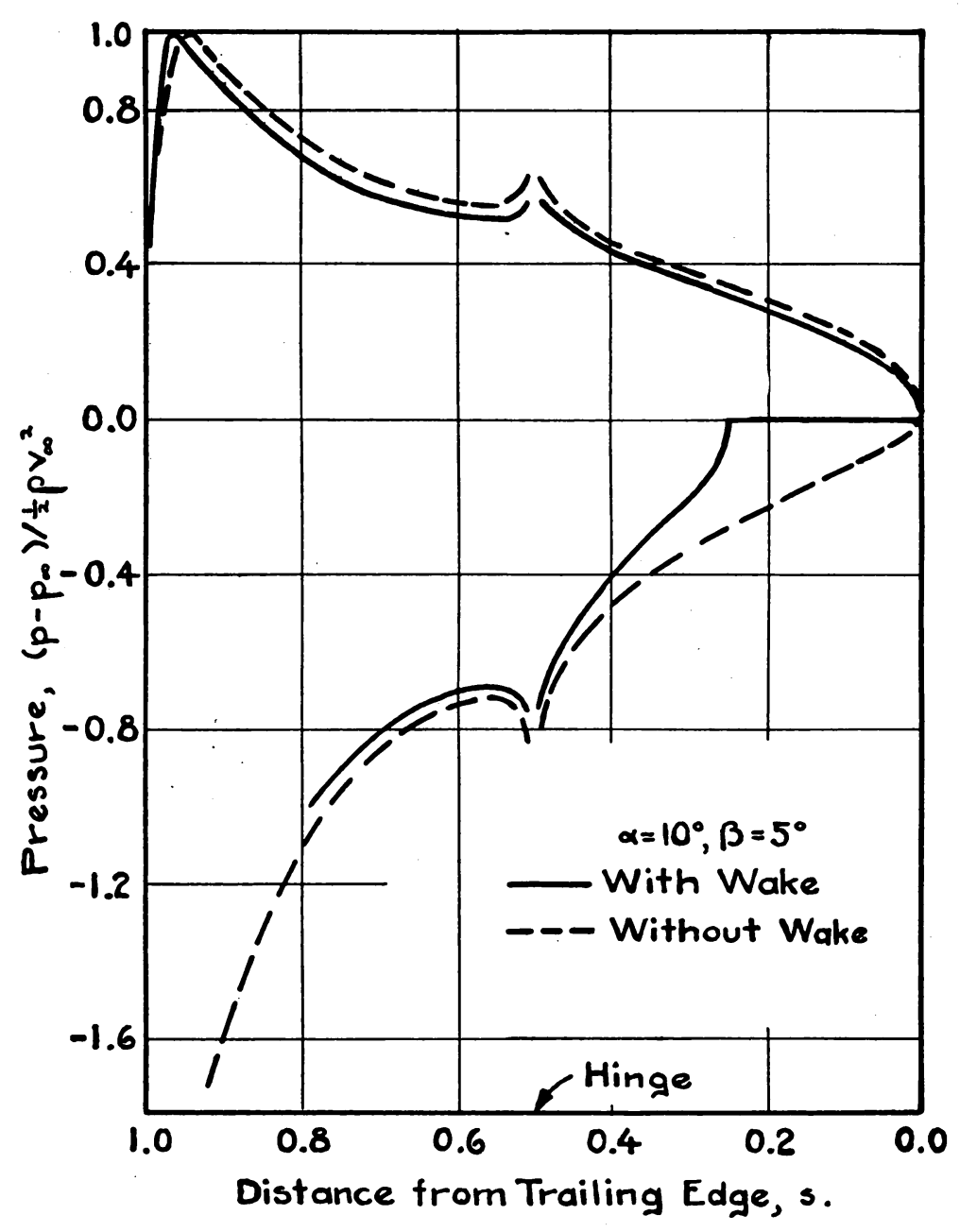

FIG. 5.

in the introduction, or some alternative. The hypothesis which we propose is a natural and direct extension of the physical assumption on the basis of which (7) was obtained. Whatever physical principle causes the velocity to 
approach the stream velocity as the distance from the airfoil becomes large might also be expected to cause it to approach the stream velocity as rapidly as possible. This is our basic hypothesis; it says that the disturbance at distant points shall be a minimum.

The general mathematical formulation of our hypothesis will be found in section 7 , and in section 8 it is shown that for our case of a broken straight line the hypothesis leads to the condition

$$
\sin \phi_{L}-\sin \phi_{S}+\frac{\beta}{\pi}\left(\sin \phi_{K}-\sin \phi_{E}\right)=0 .
$$

For $\beta=0$ this reduces to the condition which Schmieden (Reference 4 ) has used for determining the size of the wake, namely $\phi_{L}=\pi-\phi_{S}$. Putting $\beta=0$ in (7) we get $\phi_{L}-\phi_{S}=\alpha$. Hence $\phi_{L}=\frac{1}{2}(\pi+\alpha)$ and $\phi_{S}=\frac{1}{2}(\pi-\alpha)$.

The four equations (5), (6), (7), and (8) will determine the four constants involved. These equations can only be solved by successive approximation. Slide rules can be constructed for solving (7) and (8). Equations (5) and (6) can be solved by trial, the integrations being performed either graphically or numerically.

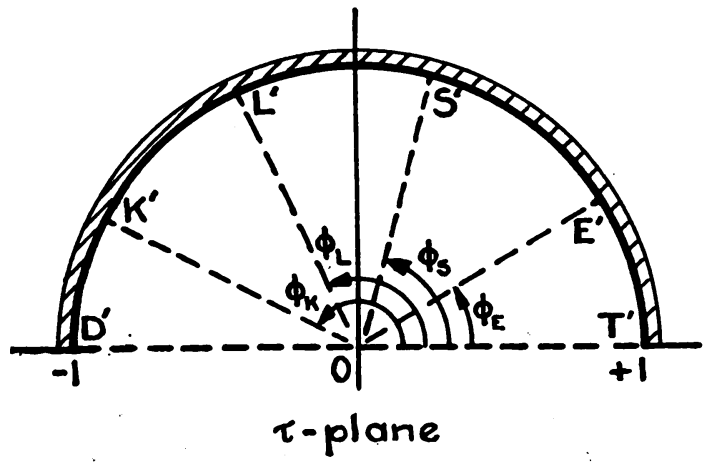

Fig. 6.

5. Proof of formulas (1) and (2) for the velocity distribution. We consider Fig. 1 as a diagram in a complex $z$-plane, $z=x+i y$. We choose the complex potential

$$
w=\psi_{1}+i \psi_{2}
$$

in such a way that the streamline $\psi_{2}=0$ will be the critical streamline which follows the surface of the airfoil and wake, with $\psi_{1}=0$ at $S$.

The $w$ plane minus the positive half of the real axis is mapped onto the interior of the upper half of the unit circle in a $\tau$ plane (Fig. 6) by the transformation (for details see, for instance, Reference 4):

$$
w=a^{2}\left(\cos \phi_{S}-\frac{1}{2}\left(\tau+\frac{1}{\tau}\right)\right)^{2}
$$


The radii from -1 to 0 and from 0 to 1 in this $\tau$-plane correspond to the free streamlines. The points on the unit circle with arguments $\phi_{E}, \phi_{S}, \phi_{L}$, and $\phi_{K}$ correspond respectively to $E, S, L, K$. The number $a$ is a constant.

Consider the complex velocity

$$
\frac{d w}{d z}=v e^{-i \theta} .
$$

Along the real axis in the $\tau$-plane between -1 and +1 the speed $v$ must be constant and equal to $v_{\infty}$, the undisturbed stream velocity. Along the successive segments of the unit circle between the points $T^{\prime}, E^{\prime}, S^{\prime}, L^{\prime}, K^{\prime}$, and $D^{\prime}$, the angle $\theta$ must have the respective constant values $-(\alpha+\beta),-\alpha, \pi-\alpha,-\alpha$ and $-(\alpha+\beta)$. It will now be shown that the following expression for the complex velocity will satisfy these conditions:

$$
\frac{d w}{d z}=v_{\infty} e^{i(\alpha+\beta)} \frac{\left(\tau-e^{-i \phi_{L}}\right) e^{i \phi_{L}}\left(\tau-e^{i \phi_{S}}\right)}{\left(\tau-e^{i \phi_{L}}\right)\left(\tau-e^{-i \phi_{S}}\right) e^{i \phi_{S}}} \cdot\left[\frac{\left(\tau-e^{-i \phi_{K}}\right) e^{i \phi_{K}}\left(\tau-e^{i \phi_{E}}\right)}{\left(\tau-e^{i \phi_{K}}\right)\left(\tau-e^{-i \phi_{E}}\right) e^{i \phi_{E}}}\right]^{\beta / \pi} .
$$

We use that determination of the bracketed quantity to the $\beta / \pi$ power whose argument vanishes for $\tau=1$. The absolute value of this expression is obviously constant and equal to $v_{\infty}$ if $\tau$ is real. For $\tau=e^{i \phi}$ on the unit circle we make use of the identity

$$
\begin{aligned}
& e^{i \phi}-e^{i \phi^{\prime}}=e^{\frac{1}{2} i\left(\phi+\phi^{\prime}\right)}\left(e^{\frac{1}{i} i\left(\phi-\phi^{\prime}\right)}-e^{-\frac{1}{i} i\left(\phi-\phi^{\prime}\right)}\right) \\
& =2 i e^{\frac{1}{4} i\left(\phi+\phi^{\prime}\right)} \sin \frac{1}{2}\left(\phi-\phi^{\prime}\right)
\end{aligned}
$$

to rewrite our expression in the form

$$
\begin{aligned}
\frac{d w}{d z}= & v_{\infty} e^{i(\alpha+\beta)} \frac{\sin \frac{1}{2}\left(\phi+\phi_{L}\right) \sin \frac{1}{2}\left(\phi-\phi_{S}\right)}{\sin \frac{1}{2}\left(\phi-\phi_{L}\right) \sin \frac{1}{2}\left(\phi+\phi_{S}\right)} \\
& \cdot\left[\frac{\sin \frac{1}{2}\left(\phi+\phi_{K}\right) \sin \frac{1}{2}\left(\phi-\phi_{E}\right)}{\sin \frac{1}{2}\left(\phi-\phi_{K}\right) \sin \frac{1}{2}\left(\phi+\phi_{E}\right)}\right]^{\beta / \pi},
\end{aligned}
$$

where the determination is chosen to agree with that in (11). If $\phi$ is between 0 and $\phi_{E}$ each factor involving the sine of a difference is negative, all the negative signs will cancel and the argument of the whole expression will be $\alpha+\beta$; hence $\theta=-(\alpha+\beta)$ as desired. If $\phi$ is between $\phi_{E}$ and $\phi_{S}$, the quantity inside the brackets will be negative and have an afgument $-\pi$; hence the entire expression will have an argument $\alpha$. In this way all the prescribed values of $\theta$ may be verified. Since our expression for $d w / d z$ is analytic and not zero inside the upper half of the unit circle in the $\tau$-plane and satisfies the required conditions for $v$ and $\theta$ on the boundary of that semicircle, it follows that the flow around our airfoil is thereby correctly determined. If we take absolute values of both sides of (12) we get the required formula (1). Also, from (9), for $\tau=e^{i \phi}$, 


$$
\begin{aligned}
\frac{d w}{d \tau} & =-a^{2}\left(\cos \phi_{S}-\frac{1}{2}(\tau+1 / \tau)\right)\left(1-1 / \tau^{2}\right) \\
& =-2 a^{2} i\left(\cos \phi_{S}-\cos \phi\right) e^{-i \phi} \sin \phi \\
& =4 a^{2} i e^{-i \phi} \sin \frac{1}{2}\left(\phi_{S}+\phi\right) \sin \frac{1}{2}\left(\phi_{S}-\phi\right) \sin \phi .
\end{aligned}
$$

Since $d z=(d z / d w) \dot{(} d w / d \tau) d \tau$, we have

$$
|d z|=\frac{1}{v}\left|\frac{d w}{d \tau}\right||d \phi| .
$$

Now $d s$ will have the same sign as $d \phi$ if $\phi$ is less than $\phi_{L}$ and opposite signs if $\phi>\phi_{L}$. We can therefore get $d s$ from $|d z|$ by removing the absolute value signs around $d \phi$ and around the quantity $\phi_{L}-\phi$ in the expression (1) for $v$. Hence (2) follows immediately on substituting (1) and (13) into (14), integrating, and setting $c^{2}=a^{2} / v_{\infty}$.

6. Proof of equation (7). We need only observe that the complex velocity must approach $v_{\infty}$ as $\tau \rightarrow 0$. Putting $\tau=0$ in (11) we get

$$
v_{\infty}=v_{\infty} e^{i(\alpha+\beta)^{\prime}} e^{-i \phi_{L}} e^{i \phi_{S}}\left[e^{-i \phi_{K}} e^{i \phi_{E}}\right]^{\beta / \pi},
$$

which reduces at once to (7).

7. Formulation of the hypothesis of minimum disturbance at distant points. Consider the function

$$
\omega(\tau)=\ln \left(v_{\infty} \frac{d z}{d w}\right)=\ln \frac{v_{\infty}}{v}+i \theta .
$$

This function is regular within the upper half of the unit circle and continuous on the diameter formed by the segment of the real axis between -1 and +1 . It is also pure imaginary on this diameter. Hence it can be continued into the lower half of the unit circle by reflection of $i \omega(\tau)$; and $\omega(\tau)$ will then be regular within the entire unit circle.

Expanding $\omega(\tau)$ in a power series we get

$$
\begin{aligned}
\frac{d w}{d z}=v_{\infty} e^{-\omega(\tau)} & =v_{\infty} e^{-\omega(0)-\omega^{\prime}(0) \tau-\omega^{\prime \prime}(0) \tau^{2} / 2-\cdots} \\
& =v_{\infty}\left\{1-\omega^{\prime}(0) \tau+\left[\left(\omega^{\prime}(0)\right)^{2}-\omega^{\prime \prime}(0)\right] \frac{\tau^{2}}{2}-\cdots\right\} .
\end{aligned}
$$

Here we have put $\omega(0)=0$, which is the condition that the velocity equals $v_{\infty}$ at infinity.

In all cases discussed in detail by Schmieden and in the present paper, the flow is uniquely determined by the condition

$$
\omega^{\prime}(0)=0 .
$$


Hence, there is one and just one flow for which

$$
\frac{d w}{d z}-v_{\infty} \sim \tau^{n} \quad \text { as } \quad \tau \rightarrow 0
$$

for $n \geqq 2$; for every other flow $n=1$. Since $\tau \rightarrow 0$ as $z \rightarrow \infty$ (for points outside the wake), $d w / d z$ will approach $v_{\infty}$ at a maximum rate as $z \rightarrow \infty$ if the flow satisfies (17). Thus (17) is, for the cases under consideration, the desired mathematical formulation of our hypothesis that the disturbance of the main stream at infinity shall be a minimum.

Equation (17) would be insufficient to determine a unique flow if one of the two points of detachment of the wake had not been determined $a$ priori. Thus in the case of an airfoil with sharp trailing edge, one point of detachment is placed at the trailing edge; for the case of a circular cylinder, the two points of detachment are assumed to be symmetrically placed. Situations can be imagined, however, such as an airfoil with blunt trailing edge, where neither point of detachment could be determined in advance. In such a case one can have a still smaller disturbance of the main stream at infinity (i.e. $n \geqq 3$ in the above asymptotic formula) by imposing in addition to (17) the condition

$$
\omega^{\prime \prime}(0)=0 .
$$

For symmetric flows this last condition is automatically satisfied whenever (17) holds.

The theory predicts the following sequence of events as one passes continuously from the case of a circular cylinder to an inclined plate by way of elliptic cylinders with increasing eccentricity but with major axis having a small constant inclination with the direction of the stream: for small eccentricities (and very high Reynolds numbers) both $\omega^{\prime}(0)$ and $\omega^{\prime \prime}(0)$ vanish, both the drag and lift are zero (see section 9), and the disturbance of the main stream at distant points is the least possible. As the ellipse becomes flatter and the trailing edge less blunt, the local viscosity and pressure conditions at the. trailing edge gain control over the lower point of detachment of the wake, the condition $\left(17^{\prime}\right)$ is lost, and a corresponding lift is developed. The flow in this state corresponds to that in the normal flight of an airfoil. For still flatter ellipses or higher angles of attack or lower Reynolds numbers, the local conditions around the leading edge gain control of the upper point of detachment, the condition (17) is also lost, and there is a positive drag proportional to $\omega^{\prime}(0)$ and a positive, although much smaller, lift. In this state the airfoil is clearly stalled. Thus the development of a lift and of a drag mark abrupt changes in the state of the flow associated with the loss of the conditions $\left(17^{\prime}\right)$ and (17) respectively; and there is a critical range of Reynolds numbers marking the effect of local conditions which determine the transitions from one type of flow to another. 
8. Proof of equation (8). Consider the expression

$$
\omega_{1}(\tau)=\ln \left(\tau-e^{i \phi_{1}}\right)-\ln \left(\tau-e^{-i \phi_{1}}\right),
$$

where $\phi_{1}$ is an arbitrary constant angle. Then

$$
\begin{aligned}
& \omega_{1}^{\prime}(\tau)=\frac{1}{\tau-e^{i \phi_{1}}}-\frac{1}{\tau-e^{-i \phi_{1}}}, \\
& \omega_{1}^{\prime \prime}(\tau)=-\frac{1}{\left(\tau-e^{\left.i \phi_{1}\right)^{2}}\right.}+\frac{1}{\left(\tau-e^{\left.-i \phi_{1}\right)^{2}}\right.}, \\
& \omega_{1}^{\prime}(0)=-e^{-i \phi_{1}}+e^{i \phi_{1}}=2 i \sin \phi_{1}, \\
& \omega_{1}^{\prime \prime}(0)=-e^{-2 i \phi_{1}}+e^{2 i \phi_{1}}=2 i \sin 2 \phi_{1} .
\end{aligned}
$$

Now from (11) it follows that $\omega(\tau)$ is the sum of a constant and constant multiples of expressions of the form $\omega_{1}(\tau)$. Hence

$$
\begin{aligned}
& \omega^{\prime}(0)=2 i\left[\sin \phi_{L}-\sin \phi_{S}+\frac{\beta}{\pi}\left(\sin \phi_{K}-\sin \phi_{E}\right)\right], \\
& \omega^{\prime \prime}(0)=2 i\left[\sin 2 \phi_{L}-\sin 2 \phi_{S}+\frac{\beta}{\pi}\left(\sin 2 \phi_{K}-\sin 2 \phi_{E}\right)\right] .
\end{aligned}
$$

Equation (8) is an immediate consequence of the first of these equations and our hypothesis (17).

9. The total drag and lift. According to the Levi-Civita formula, the total drag on the airfoil is proportional to $\omega^{\prime}(0)$. From our hypothesis (17) it follows that the drag is zero. In fact, in the case under consideration our hypothesis is equivalent to the condition that the drag vanishes. However, in other situations such as the case of a finite wake discussed by Kolscher (Reference 2), the two conditions differ.

The total lift may be calculated from the Levi-Civita formula

$$
Y=\frac{1}{4} \pi \rho v_{\infty}^{2} c^{2} i \omega^{\prime \prime}(0), \quad c^{2}=a^{2} / v_{\infty}
$$

and (18) to be

$$
Y=-\frac{1}{2} \pi \rho v_{\infty}^{2} c^{2}\left[\sin 2 \phi_{L}-\sin 2 \phi_{S}+\frac{\beta}{\pi}\left(\sin 2 \phi_{K}-\sin 2 \phi_{E}\right)\right] .
$$

For $\beta=0$ this reduces to the Schmieden expression

$$
Y=\pi \rho v_{\infty}^{2} c^{2} \sin \alpha
$$


10. The concentrated forces at $L$ and $K$. Consider the flow around a corner $K$ with arbitrary angle $\mu \pi, 0 \leqq \mu \leqq 1$. Draw the small circle $C$ of radius $r$ about $K$. The finite force at $K$, if any, will be obtained by applying Euler's momentum theorem to the fluid inside $C$ and taking the limit as $r$ approaches
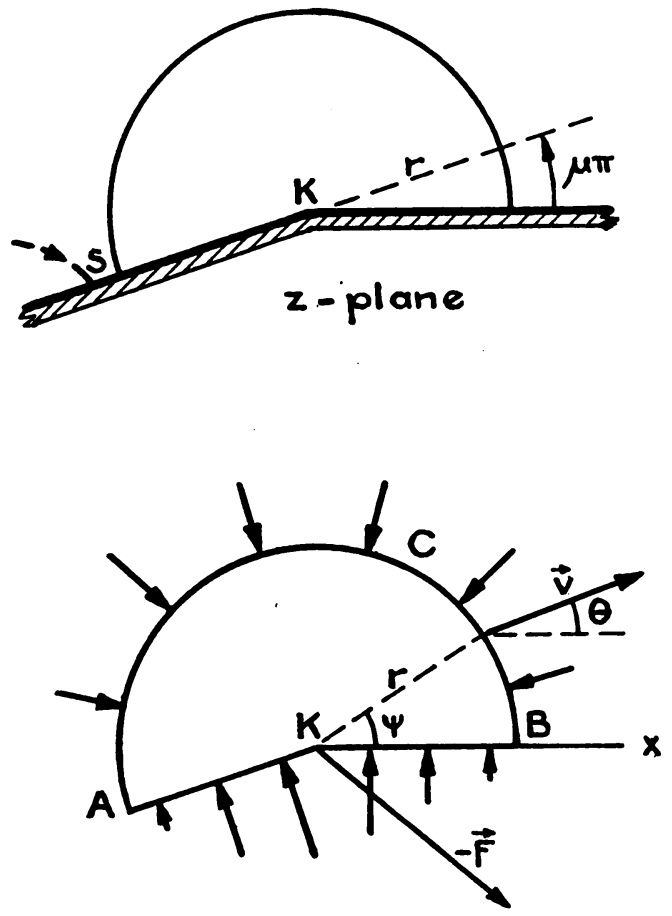

FIG. 7.

zero. In Fig. 7 we show the fluid as a free body, where $-\vec{F}$ is the reaction of the concentrated force $\vec{F}$ on $K$.

We have

$$
\vec{F}-\int_{C+A K B} \vec{n} p d s=\rho \int_{C} \vec{n} \cdot \vec{v} \vec{v} d s,
$$

where $\vec{n}$ is the inward unit normal.

It is assumed that the stagnation point $S$ is not at the corner. Hence for small $r$, setting $\lambda=\mu /(1+\mu), 0 \leqq \lambda \leqq \frac{1}{2}$,

$$
\frac{d w}{d z}=k /(z-K)^{\lambda}+0\left(r^{1-2 \lambda}\right)
$$

where $k$ is a constant and $0\left(r^{1-2 \lambda}\right)$ is a quantity such that $0\left(r^{1-2 \lambda}\right) / r^{1-2 \lambda}$ is bounded as $r \rightarrow 0$. Hence if we put $z-K=r e^{i \psi}$, 


$$
\begin{aligned}
v & =|k| r^{-\lambda}+0\left(r^{1-2 \lambda}\right), & & v^{2}=|k|^{2} r^{-2 \lambda}+0\left(r^{1-3 \lambda}\right), \\
e^{i \theta} & =(|k| / k) e^{i \lambda \psi}+o\left(r^{1-2 \lambda}\right), & p & =-\frac{1}{2}|k|^{2} \rho r^{-2 \lambda}+0\left(r^{\sigma}\right),
\end{aligned}
$$

where $\sigma=0$ if $\lambda \leqq 1 / 3,=1-3 \lambda$ if $\lambda>1 / 3$, and $o\left(r^{1-2 \lambda}\right)$ is a quantity such that $o\left(r^{1-2 \lambda}\right) / r^{1-2 \lambda} \rightarrow 0$ as $r \rightarrow 0$.

Now

$$
\int_{A}^{K} n p d s=-\frac{1}{2} \vec{n}|k|^{2} \rho \int_{0}^{r} r^{-2 \lambda} d r+\vec{n} \int_{0}^{r} 0\left(r^{\sigma}\right) d r .
$$

Both these integrals tend to zero with $r$ if $\mu<1$. If $\mu=1$, this integral and the corresponding integral along $K B$ both diverge, but since, in this case, the pressures along $A K$ and $K B$ are directly opposing, it is permissible to use the principal value of the integral along $A K B$. With this understanding we may write

$$
\int_{A K B} \vec{n} p d s=-\frac{1}{2} \vec{n}_{1}|k|^{2} \rho \int_{0}^{r}\left(r^{-1}-r^{-1}\right) d r+\int_{0}^{r} \vec{n} 0\left(r^{\sigma}\right) d r,
$$

where $\vec{n}_{1}$ is the normal to $A K$. Both these integrals tend to zero. For the arc $C$,

$$
\int_{C} \vec{n} p d s=-\frac{1}{2}|k|^{2} \rho \int_{0}^{\pi(1+\mu)} r^{-2 \lambda+1} e^{i(\psi-\pi)} d \psi+\int_{C} \vec{n} 0\left(r^{\sigma}\right) d s .
$$

These integrals also approach zero; the first integral is zero if $\mu=1$.

The Euler momentum theorem thus reduces to

$$
\vec{F}=\lim _{r \rightarrow 0} \rho \int_{C} \vec{n} \cdot \vec{v} \vec{v} d s
$$

But $\cos (\vec{n}, \vec{v})=\cos (\psi-\theta-\pi), \vec{v}=v e^{i \theta}$,

$$
\rho \int_{C} \vec{n} \cdot \vec{v} \vec{v} d s=-\frac{\rho}{2} \int_{C} v^{2} e^{i \psi} d s-\frac{\rho}{2} \int_{C} v^{2} e^{-i \psi} e^{2 i \theta} d s .
$$

The first of these integrals is similar to (20) and will approach zero for the same reason. For the second integral,

$$
-\frac{\rho}{2} \int_{C} v^{2} e^{-i \psi} e^{2 i \theta} d s=\frac{-\rho}{2} \int_{0}^{\pi(1+\mu)} \frac{|k|^{2}}{r^{2 \lambda}} e^{-i \psi} \frac{|k|^{2}}{k^{2}} e^{2 i \lambda \psi} r d \psi-\frac{\rho}{2} \int_{C} o\left(r^{1-4 \lambda}\right) d s .
$$

The last integral approaches zero. The first integral also approaches zero if $\mu<1$. If $\mu=1$, however, its value is not zero. Evaluating this integral we get finally

$$
\begin{aligned}
\vec{F} & =-\pi \rho \frac{|k|^{4}}{k^{2}} & \text { if } & \mu=1, \\
& =0 & \text { if } & \mu \neq 1 .
\end{aligned}
$$


We have thus proved the general result that for a concentrated force to exist at a corner which is not a stagnation point the corner must be a cusp. In particular, there will be no concentrated force at the hinge $K$. The concentrated force at the leading edge may be found by evaluating $k$, giving $\vec{F}=-4 \rho \pi v_{\infty}^{2} c^{2} e^{-i \alpha} \sin ^{2} \phi_{L} \sin ^{2} \frac{1}{2}\left(\phi_{L}-\phi_{S}\right)\left[\frac{\sin \frac{1}{2}\left(\phi_{L}+\phi_{K}\right) \sin \frac{1}{2}\left(\phi_{L}-\phi_{E}\right)}{\sin \frac{1}{2}\left(\phi_{K}-\phi_{L}\right) \sin \frac{1}{2}\left(\phi_{L}+\phi_{E}\right)}\right]^{\beta / \pi}$,

where, as before, $c^{2}=a^{2} / v_{\infty}$. The direction of the force is parallel to the plate $L K$ and toward the left. In the case $\beta=0$ this reduces to

$$
\vec{F}=-\rho \pi v_{\infty}^{2} c e^{2} e^{-i \alpha} \sin ^{2} \alpha,
$$

which agrees with the expression given by Schmieden (Reference 4).

11. The width of the wake. It will now be shown that under the minimum hypothesis (17) the width of the wake tends to zero asymptotically as the distance from the airfoil becomes infinite.

We have as in (16)

$$
\frac{d z}{d w}=\frac{1}{v_{\infty}} e^{\omega(\tau)}=\frac{1}{v_{\infty}}\left[1+\omega^{\prime \prime}(0) \frac{\tau^{2}}{2}+\omega^{\prime \prime \prime}(0) \frac{\tau^{3}}{6}+\cdots\right] .
$$

Also

$$
\frac{d w}{d \tau}=-a^{2}\left(\frac{1}{2} \frac{1}{\tau^{3}}-\frac{1}{\tau^{2}} \cos \phi_{S}+\cos \phi_{S}-\frac{1}{2} \tau\right) .
$$

Hence

$$
d z=\frac{d z}{d w} \frac{d w}{d \tau} d \tau=-\frac{a^{2}}{v_{\infty}}\left[\frac{1}{2} \frac{1}{\tau^{3}}-\frac{1}{\tau^{2}} \cos \phi_{S}+\frac{\omega^{\prime \prime}(0)}{4} \frac{1}{\tau}+0(1)\right] d \tau .
$$

Integrating :

$$
z=C_{1}+i C_{2}-\frac{a^{2}}{v_{\infty}}\left[-\frac{1}{4} \frac{1}{\tau^{2}}+\frac{1}{\tau} \cos \phi_{S}+\frac{\omega^{\prime \prime}(0)}{4} \ln \tau+0(\tau)\right],
$$

where $C_{1}$ and $C_{2}$ are real constants. This expression is of the form

$$
z=C_{1}+i C_{2}+\frac{A}{\tau^{2}}+\frac{B}{\tau}+i D \ln \tau+0(\tau)
$$

where $A, B$, and $D$ are real constants and $A$ is positive. For $\tau$ real and positive this will give the coordinates of a point $\left(x_{1}, y_{1}\right)$ on the lower boundary of the wake:

$$
\begin{aligned}
& x_{1}=C_{1}+\frac{A}{\tau^{2}}+\frac{B}{\tau}+0(\tau), \\
& y_{1}=C_{2}+D \ln \tau+0(\tau) .
\end{aligned}
$$


If $\tau$ is negative we put $\tau=-\tau^{\prime}$ where $\tau^{\prime}$ is positive and obtain in like manner the coordinates $\left(x_{2}, y_{2}\right)$ of a point on the upper boundary of the wake:

$$
\begin{aligned}
& x_{2}=C_{1}+\frac{A}{\tau^{\prime 2}}-\frac{B}{\tau^{\prime}}-\pi D+0\left(\tau^{\prime}\right), \\
& y_{2}=C_{2}+D \ln \tau^{\prime}+0\left(\tau^{\prime}\right) .
\end{aligned}
$$

Consider the particular values of $\tau$ and $\tau^{\prime}$ defined by the equations

$$
\begin{aligned}
\frac{1}{\tau} & =\frac{-B+\sqrt{B^{2}-4 A\left(C_{1}-t\right)}}{2 A}, \\
\frac{1}{\tau^{\prime}} & =\frac{B+\sqrt{B^{2}-4 A\left(C_{1}-\pi D-t\right)}}{2 A},
\end{aligned}
$$

where $t$ is a large real positive parameter. As $t$ becomes infinite both $\tau$ and $\tau^{\prime}$ tend to zero through positive values. Substituting these expressions into (22) and (23) we get

$$
\begin{aligned}
& x_{2}-x_{1}=0\left(\tau^{\prime}\right)-0(\tau), \\
& y_{2}-y_{1}=D \ln \frac{-B+\sqrt{B^{2}-4 A\left(C_{1}-t\right)}}{B+\sqrt{B^{2}-4 A\left(C_{1}-\pi D-t\right)}}+0\left(\tau^{\prime}\right)-0(\tau) .
\end{aligned}
$$

As $t \rightarrow \infty$ both these differences approach zero; hence the width of the wake tends to zero.

12. Details of the calculations. In the numerical calculation of pressure distributions, the evaluation of the constants is the most time consuming. As mentioned previously, it is a simple matter to construct slide rules for Eqs. (7) and (8). It was also found to save time to evaluate the integrals in (5) and (6) graphically. Templates were constructed for the functions $\log \sin \phi, \log \sin \phi / 2$, and $\beta / \pi \log \sin \phi / 2$. By properly adjusting the phase, one can quickly draw from these templates the curves $\log \sin \frac{1}{2}\left(\phi-\phi_{L}\right)$, $\log \sin \frac{1}{2}\left(\phi+\phi_{L}\right), \beta / \pi \log \sin \frac{1}{2}\left(\phi-\phi_{E}\right)$, etc. By addition and subtraction of the ordinates thus obtained one gets the logarithm of the integrand, and hence the value of the integrand itself from a graph of the logarithm function. The integral can then be found either graphically or by means of a planimeter. The pressure distribution shown in Fig. 5 and the associated points in Figs. 3 and 4 were calculated in this way.

If the integrals in (5) and (6) are computed numerically, the infinite discontinuities of the integrand at $\phi_{E}$ and $\phi_{K}$ are troublesome. There is not actually a discontinuity at $\phi_{K}$, but the integrand goes to zero so abruptly near this point that it is convenient to treat the point as if it were a discontinuity. The usual method of "subtracting off" the discontinuity is unsatis- 
factory because the result will be an abrupt zero of the type just mentioned. The following procedure was used as an alternative: after isolating the discontinuity (say at $\left.\phi_{1}\right)$ at the center of a small interval $\left(\phi_{1}-\delta, \phi_{1}+\delta\right)$, and approximating the sine of an angle by the angle, the integrand reduces to the form $g(\phi)\left|\phi-\phi_{1}\right|^{\nu}$, where $\nu$ is some constant and $g(\phi)$ is continuous (and smooth). A linear transformation will change this integral into one of the form $\int_{-1}^{+1} \bar{g}\left(\phi^{\prime}\right)\left|\phi^{\prime}\right| \nu d \phi^{\prime}$. One can then approximate $\bar{g}\left(\phi^{\prime}\right)$ by a quadratic function of the form $a \phi^{\prime 2}+b \phi^{\prime}+c$ by interpolating to the values of $\bar{g}$ at $-1,0$, and +1 ; and the resulting integral is easily computed. The final result is a formula for the original integral in terms of the values of $g$ at $\phi_{1}-\delta, \phi_{1}$, and $\phi_{1}+\delta$ which is strictly analogous to, and in fact is a generalization of, Simpson's rule. 